\title{
Error-Control for Compressed Sensing of Images with Multi-Channel Transmission
}

\author{
Hsiang-Cheh Huang \\ Department of Electrical \\ Engineering \\ National University of Kaohsiung \\ Kaohsiung 811, Taiwan, R.O.C. \\ hch.nuk@gmail.com
}

\author{
Ting-Hsuan Wang \\ Department of Electrical \\ Engineering \\ National University of Kaohsiung \\ Kaohsiung 811, Taiwan, R.O.C. \\ hch.nuk@gmail.com
}

\author{
Feng-Cheng Chang \\ Department of Innovative \\ Information and Technology \\ Tamkang University \\ Ilan 262, Taiwan, R.O.C. \\ 135170@mail.tku.edu.tw
}

\begin{abstract}
Compressed sensing has attracted much attention in researches due to its new thoughts and superior performances in data compression. For the delivery of compressed information, because it is vulnerable to channel errors during transmission, error control for compressed information has long been a practical topic for researches and applications. In this paper, we aim at the error control of compressed sensing of images. With compressed sensing, very few amounts of coefficients are capable of reconstructing the image with reasonable quality. For the delivery of compressively sensed coefficients over independent and lossy channels, reconstructed image with reasonable quality over a variety of lossy rates can be obtained. Simulation results have pointed out that with the proposed algorithm, the applicability and superiority in performances can be acquired over conventional algorithm in this field.
\end{abstract}

Keywords-compressed sensing; error control; image quality; transmission

\section{INTRODUCTION}

Data compression has long been an important topic in the field of signal processing. With the widely use of smartphone cameras or tablets, vast amounts of multimedia contents, mostly images, have accumulated drastically. Thus, how to efficiently perform data compression on the multimedia contents would be in urgent needs. There have been successful and popular standards for image compression, including the well-known JPEG, which employs discrete cosine transform (DCT), and JPEG 2000, which applies discrete wavelet transform (DWT), for compression. With the evolution of new techniques, advancements in data compression can also be expected, and compressed sensing presents some novelties over its predecessors.

Compressed sensing [1] [2] is a newly developed branch in data compression researches in the last couple of years. It requires the sampling rate, which is far less than the Nyquist rate, with the capability of reconstructing the original signal to be above some acceptable level. After performing compression at the encoder, compressed signals need to be transmitted to the decoder over lossy channels. Finally, at the decoder, received signals need to be reconstructed to be as resemble as its counterpart at the encoder. Besides the compression capabilities, in this paper, we aim at the error control of compressively sensed signals for transmitting over lossy channels. For transmitting the compressively sensed signals over multiple independent channels, reconstructed quality presents much better than those delivered over the single-channel transmission. Besides looking for compression performances and error control capabilities, compressed sensing can extend to relating topics, such as image compression [3][4], theoretic derivations [5], and information hiding [6][7].

This paper is organized as follows. In Sec. II, we briefly describe the fundamentals and mathematical representations of compressed sensing. In Sec. III, we present the proposed method for transmitting compressively sensed signals over independent and lossy channels. Simulation results are demonstrated in Sec. IV, which point out the vulnerability of compressively sensed signals for the transmission over a single channel, and the alleviation of image quality degradation with our algorithm for multiple channel transmission. Finally, we address the conclusion of this paper in Sec. V.

\section{FUNDAMENTAL DESCRIPTIONS OF COMPRESSED SENSING}

Compressed sensing, abbreviated as CS, aims at looking for new sampling scheme that goes against conventional sampling theorem, or the widely acquainted NyquistShannon theorem. With CS, a much smaller rate than twice the maximal bandwidth can be achieved to meet perfect recovery of reconstruction.

In compressed sensing, it is composed of the sparsity principle, and the incoherence principle [1][2], described as follows.

$>$ For the sparsity principle, it implies the information rate in data compression. In compressive sampling, it is expected to be much smaller than the sampling rate required, and can be represented with the proper basis $\Psi, \Psi \in C^{N \times N}$, and $C$ means the complex number. More specifically, $\Psi$ is the basis to reach sparsity with a $k$-sparse coefficient vector $\mathbf{x}, \mathbf{x} \in C^{N \times 1}$, with the condition that

$$
\mathbf{f}=\Psi \mathbf{x} \text {. }
$$


Here, $\mathbf{f}$ denotes the reconstruction corresponding to the original signal.

For the incoherence principle, it extends the duality between time and frequency. The measurement basis $\Phi, \Phi \in C^{m \times N}$, which acts like noiselet, is employed for sensing the signal $\mathbf{f}$, with the condition that

$$
\mathbf{y}=\Phi \mathbf{f}
$$

Here, $\mathbf{y}$ denotes the measurement vector. We note that Eq. (2) is an underdetermined system.

Considering Eq. (1) and Eq. (2), by minimizing the $l_{1}$ norm of $\mathbf{x}$, i.e., $\min \|\mathbf{x}\|_{1}$, subject to $\Phi \Psi \mathbf{x}=\mathbf{y}$, compressed sensing guarantees the perfect recovery with probability close to 1.0. Besides, because of looking for $\min \|\mathbf{x}\|_{0}$, or $l_{0^{-}}$ minimization is an NP-hard combinatorial problem, we look for $l_{1}$-minimization instead.

\section{PROPOSED AlgORITHM}

From the past experiences in data compression, due to the fact that compressed multimedia contents are vulnerable to channel errors, error-controlled transmission would be required [8]. In this paper, during delivery from the encoder to the decoder, we employ the concept of multi-channel transmission to alleviate the degradation of reconstructed image quality. Here, the channels imply the packet-loss channel with the provided packet loss rate $p_{e}$, with the subscript $e$ denoting the error induced during transmission. In the multiple-channel scenario, each channel is independent with the other channels.

We describe the delivery of compressed sensing coefficients and propose our algorithm for error-controlled transmission as follows.

\section{A. Image Compression with Compressed Sensing}

In this paper, we employ the test image, airport, with size of $1024 \times 1024$, in Fig. 1. We use large test images to show the performances of CS.

By following [3], for compressing with CS, we choose $K_{1}$ $=4,000$ coefficients in $\Psi$, and $K_{2}=80,000$ coefficients in $\Phi$, from the $1024 \times 1024=1,048,576$ pixels in the original image. With this setting, compression ratio of 262 times can be reached. Figure 2 presents the reconstruction with CS coefficients after decompression, with the peak signal-tonoise ratio (PSNR) of $25.159 \mathrm{~dB}$. Subjective and objective qualities in Fig. 2 serve as the baseline for the comparison with the following simulations for lossy transmission.

\section{B. Transmission of Compressed Sensing Coefficients over Lossy Channels}

Based on Eq. (2), compressed sensing coefficients are ready for transmitting over lossy channels. We set the loss rate $p_{e}=$ 0.25 for convenience, meaning that $25 \%$ of the compressed sensing coefficients may be lost during delivery.

Figure 3 demonstrates the resulting performance after experiencing $25 \%$ of loss rate, causing the PSNR of 17.370 $\mathrm{dB}$. Degradations can be easily observed by comparisons between Fig. 3 and Fig. 2.

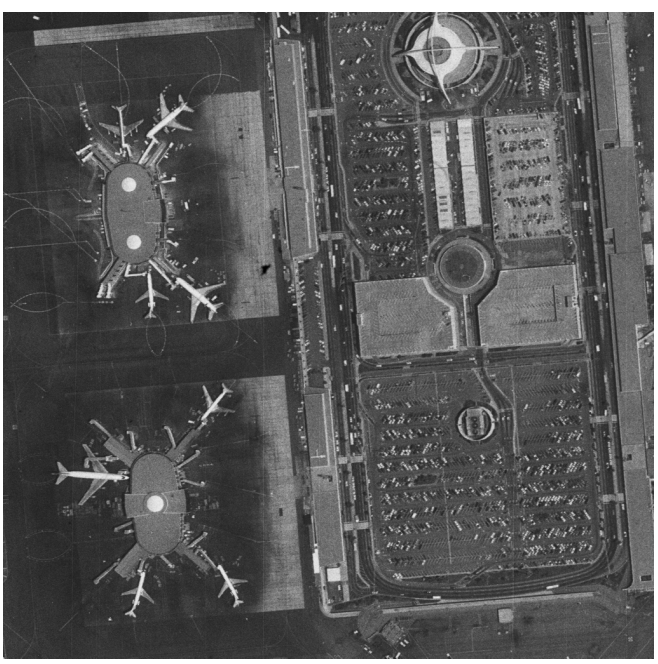

Figure 1. Test image of airport, with size of $1024 \times 1024$.

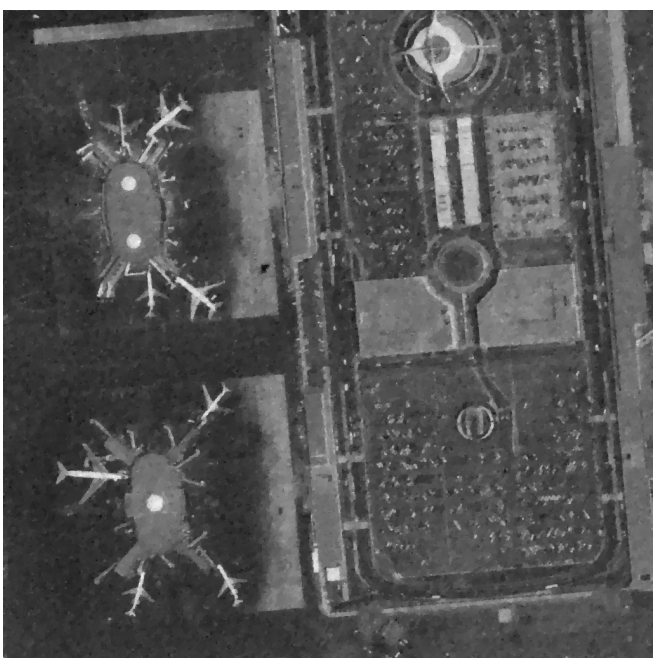

Figure 2. Compressed sensing of Fig. 1 with $K_{1}=4,000$ and $K_{2}=80,000$. Resulting PSNR $=25.159 \mathrm{~dB}$.

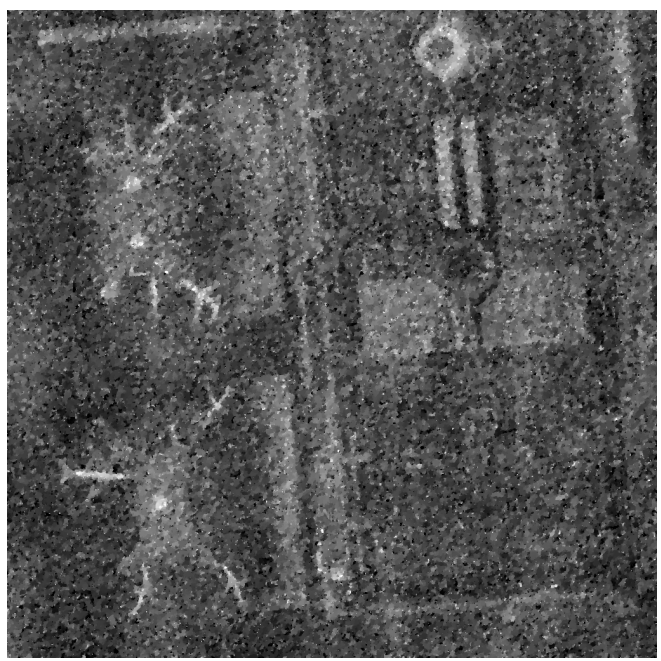

Figure 3. Compressively sensed image of Fig. 1 with $K_{1}=4,000$ and $K_{2}=$ 80,000 . Loss rate $p_{e}=0.25$. Reconstructed PSNR $=17.370 \mathrm{~dB}$. 


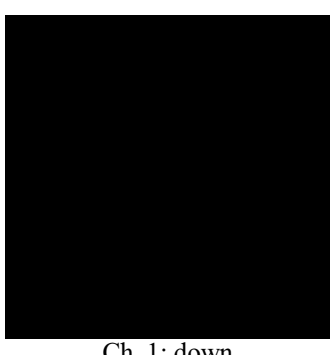

Ch. 1: down

Ch. 3: $23.582 \mathrm{~dB}$

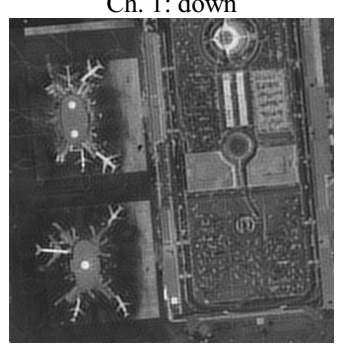

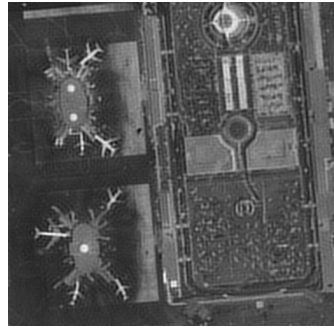

Ch. 2: $23.596 \mathrm{~dB}$

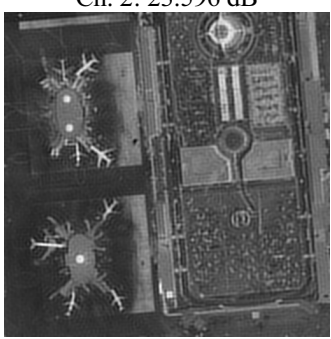

Ch. $4: 23.559 \mathrm{~dB}$
Figure 4. Transmission over multiple independent channels, with $K_{1}=$ 1,000 and $K_{2}=20,000$ for each channel. Suppose one of four channels, or Channel 1 , is down, and Channels 2 to 4 are alive. Total loss rate is 0.25 .

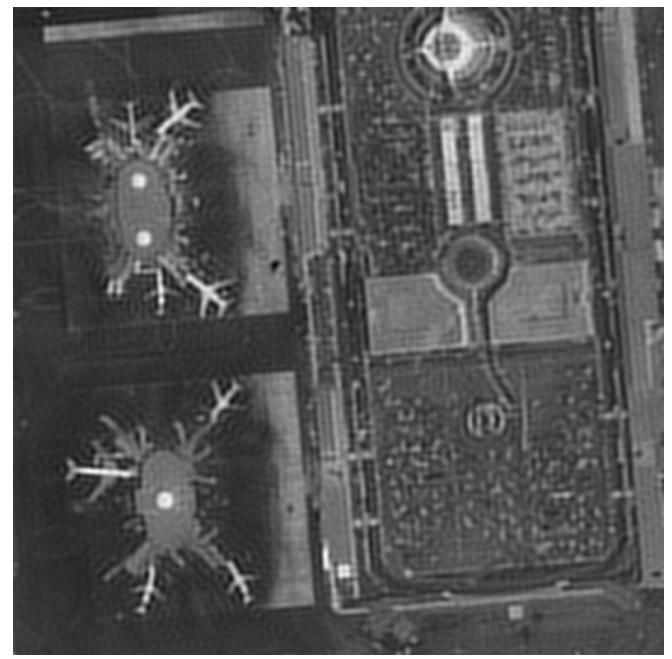

Figure 5. Recovery of coefficients in Ch. 1 by taking the median from corresponding channels, and reconstruct the image from recovered $\mathrm{Ch} .1$ coefficients and received coefficients from other channels. Reconstructed quality in PSNR $=23.467 \mathrm{~dB}$.

\section{Recovery of CS Coefficients and Reconstruction of Image}

For transmission over lossy channels, every compressed sensing coefficient experiences the loss rate $p_{e}$, and it may be lost during transmission. To alleviate the reconstruction quality as depicted in Fig. 3, CS coefficients may be transmitted sequentially over multiple channels. Due to the high correlations between sub-sampled originals, CS coefficients tend to be similar at the same transmission order. Once the coefficient is lost, it should be recovered from corresponding coefficients in other channels.

For simplicity, we split the original image into four subsampled images; each one experiences the compression with compressed sensing. For keeping the compression ratio, we choose $K_{1}=1,000$ and $K_{2}=20,000$ for each sub-sampled image with the size of $512 \times 512$, which corresponds to $25 \%$ of that in Fig. 2. Suppose that there are four channels for transmission in this paper. Then, coefficients corresponding to each sub-sampled images are transmitted over four independent channels. Suppose Channel 1 is down, and Channels 2 to 4 are alive, which also results in the loss rate of 0.25 . In Fig. 4, we demonstrate the scenarios described above. For making up the lost coefficients in Channel 1, we take the median value of corresponding coefficients in Channels 2, 3, and 4 to replace the lost coefficient in Channel 1. Recovered image is depicted in Fig. 5, with the PSNR $=23.467 \mathrm{~dB}$. We can easily observe that the quality in Fig. 5 is much better than that in Fig. 3. Moreover, due to channel loss, even the reconstruction scheme is applied, the reconstructed quality in Fig. 5 is still a bit inferior to that in Fig. 2. With the scenario presented, reconstruction of lost coefficients may be predicted from the correctly received coefficients from other channels.

Due to the randomness in the packet-loss channels, it would not be as easy as reconstructing the scenario in Fig. 4. Still, we make comparisons with the coefficients at the same position in the four channels. We first sort the magnitudes of the four received coefficients in decreasing order. And there are several cases that are possible for the recovery of CS coefficients. We omit the case that four coefficients are received correctly because no reconstruction is necessary.

$>$ Suppose one coefficient among the four is lost in this case. If the smallest magnitude (or the fourth coefficient) is smaller than some threshold (for example, $5 \%$ ) of the magnitude of the third coefficient, the fourth coefficient is recovered by taking the median of the remaining three coefficients.

$>$ Suppose two coefficients among the four are lost in this case. When the magnitude of the third coefficient is smaller than $5 \%$ of the second one, we assume that two coefficients are lost. The lost coefficients are replaced by the median of the first and second coefficients.

$>$ Suppose three coefficients among the four are lost in this case. All the lost coefficients are replaced by the first coefficient.

$>$ Suppose all the four coefficients are lost in this case if the magnitude of the first coefficient lie below some small value around zero. No reconstruction is applicable.

With our algorithm, we expect to obtain enhanced performances with multiple-channel transmission than those with single-channel transmission, as depicted in Fig. 3.

\section{Simulation Results}

In our simulations, as we stated before, we choose the test image of airport, with the picture sizes of $1024 \times 1024$, for conducting simulations. As we noted in Sec. III, in each sub-sampled image, we set $K_{1}=1000$ compressed sensing coefficients, and $K_{2}=2000$ for noiselets for keeping the compression ratio. 


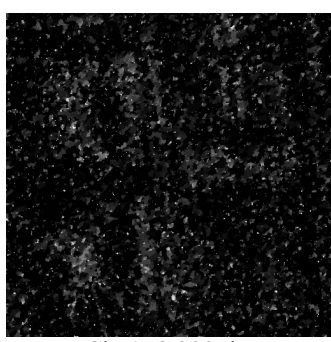

Ch. $1: 8.392 \mathrm{~dB}$

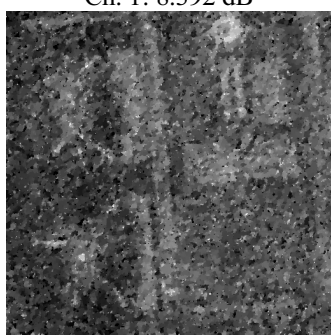

Ch. 3: $17.458 \mathrm{~dB}$

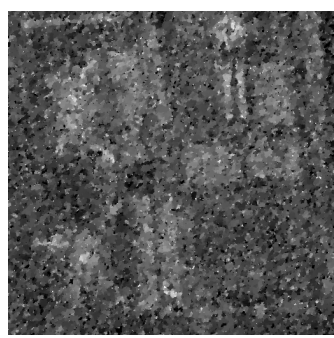

Ch. 2: $16.864 \mathrm{~dB}$

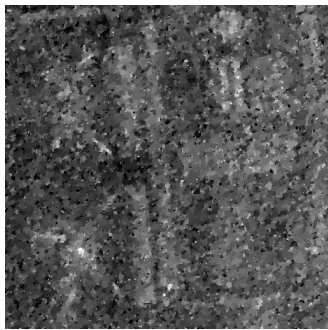

Ch. 4: $17.091 \mathrm{~dB}$
Figure 6. Transmission over multiple independent channels. Loss rate $p_{e}$ $=0.25$ for each channel. Results in Channel 1 performs inferior because coefficients with large magnitudes are lost due to channel errors.

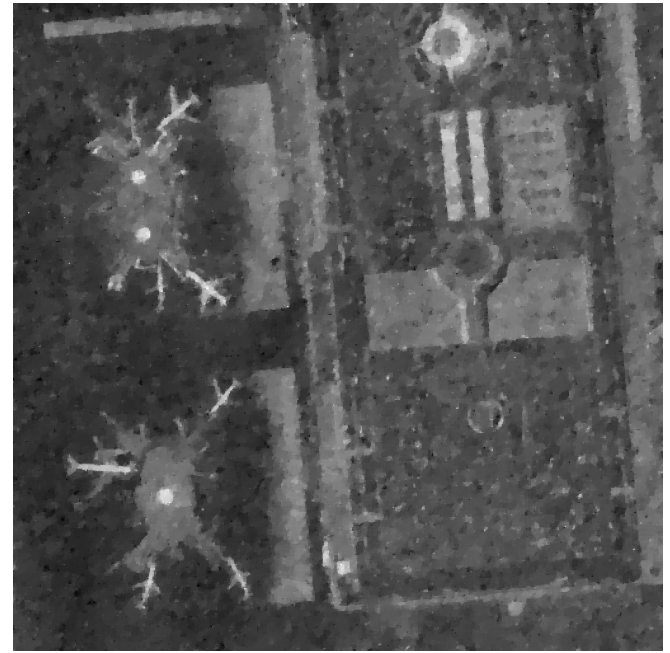

Figure 7. Recovery of coefficients by taking the median from correctly received channels. Reconstructed quality in PSNR $=22.044 \mathrm{~dB}$.

We split the original image into four sub-sampling images with the size of $512 \times 512$, and each of them is transmitted over independent lossy channels with $p_{e}=0.25$ for the ease of comparisons with Fig. 4. Simulation results are depicted in Fig. 6. For the received image in Channel 1, because the large magnitude coefficients may be lost during transmission, severe degradation can be observed. For the reconstructed sub-sampled images from Channels 2 to 4 , they result in similar qualities. With the reconstruction schemes described in Sec. III.C, reconstructed image can be recovered by taking the median from the correctly received channels in Fig. 7. We can also make comparisons between the results in Fig. 5 and Fig. 7. Reconstructed quality in Fig. 5 presents better than that in Fig. 7 even when the loss rate are 0.25 for the two cases. On the one hand, because we set the condition that Channel 1 is down in Fig. 5, reconstructed coefficients can be correctly recovered. On the other hand, because we apply random loss for all coefficients among four channels, erroneous detection of lost coefficients may be expected, which leads to the degradation in image quality. From the results presented above, proposed algorithm point out the applicability for transmitting over lossy channels.

\section{CONCLUSIONS}

In this paper, we observed the vulnerability of compressively sensed information for transmission over lossy channels, and proposed our algorithm to transmit compressed information over multiple independent and lossy channels. Based on the experiences in the field of data compression, there is the need for protecting compressed coefficients, including compressively sensed ones, from channel errors for transmitting over lossy channels. There are high correlations between sub-sampled images in the original image. By use of transmitting compressively sensed coefficients from subsampled images, lost coefficients have the possibility to be recovered by use of taking the median from the correctly received coefficients from other channels. Simulation results have presented the enhanced performances with multiple channel transmission over single channel transmission of compressively sensed coefficients. We are going to look for other effective means to ensure the error-controlled transmission for compressed sensing of images.

\section{ACKNOWLEDGMENT}

The authors would like to thank Ministry of Science and Technology (formerly National Science Council), Taiwan, R.O.C., for supporting this paper under Grant No. NSC1022220-E-390-002.

\section{REFERENCES}

[1] E. J. Candes and M. B. Wakin, "An introduction to compressive sampling," IEEE Signal Processing Magazine, vol. 25, no. 2, pp. 2130, Mar. 2008.

[2] T. Arildsen and T. Larsen, "Compressed sensing with linear correlation between signal and measurement noise," Signal Processing, vol. 98, pp. 275-283, May 2014.

[3] J. Romberg "Imaging via compressive sampling," IEEE Signal Processing Magazine, vol. 25, no. 2, pp. 14-20, Mar. 2008.

[4] C. Deng, W. Lin, B. S. Lee, and C. T. Lau, "Robust image coding based upon compressive sensing," IEEE Trans. Multimedia, vol. 14, no. 2, pp. 278-290, 2012.

[5] W. Jiang and J. Yang, "The rate-distortion optimized quantization algorithm in compressive sensing," Optik - International Journal for Light and Electron Optics, online available (DOI: 10.1016/j.ijleo.2014.01.147)

[6] H. C. Huang and F. C. Chang, "Robust image watermarking based on compressed sensing techniques," Journal of Information Hiding and Multimedia Signal Processing, vol. 5, no. 2, pp. 275-285, Apr. 2014.

[7] W. Li, J. S. Pan, L. J. Yan, C. S. Yang, and H. C. Huang, "Data hiding based on subsampling and compressive sensing," Proc. Int'l Conf. on Intelligent Information Hiding and Multimedia Signal Processing, pp. 611-614, 2013.

[8] H. C. Huang and H. M. Hang, "Error control for low bit rate coding transmission," Computer Standards \& Interfaces, vol. 20, no. 6-7, pp. 404, Mar. 1999. 